\title{
PROJEKT ZENTRALDEPOT DER UNIVERSITÄTS- UND LANDESBIBLIOTHEK TIROL
}

\section{von Christian Kössler}

Seit 2003 fungierte eine zentrale Depotbibliothek im Westen Innsbrucks als zusätzlicher Standort für Printmedien-Bestände. Auf einer Fläche von $3.000 \mathrm{~m}^{2}$ standen dort insgesamt 17.500 lfm Bibliotheksregale zur Verfügung.

Platzmangel und ein auslaufender Mietvertrag begründeten die Suche nach einer entsprechenden Alternative, die mit dem wenige Kilometer südlich von Innsbruck gelegenen Standort „Gärberbach“ gefunden werden konnte.

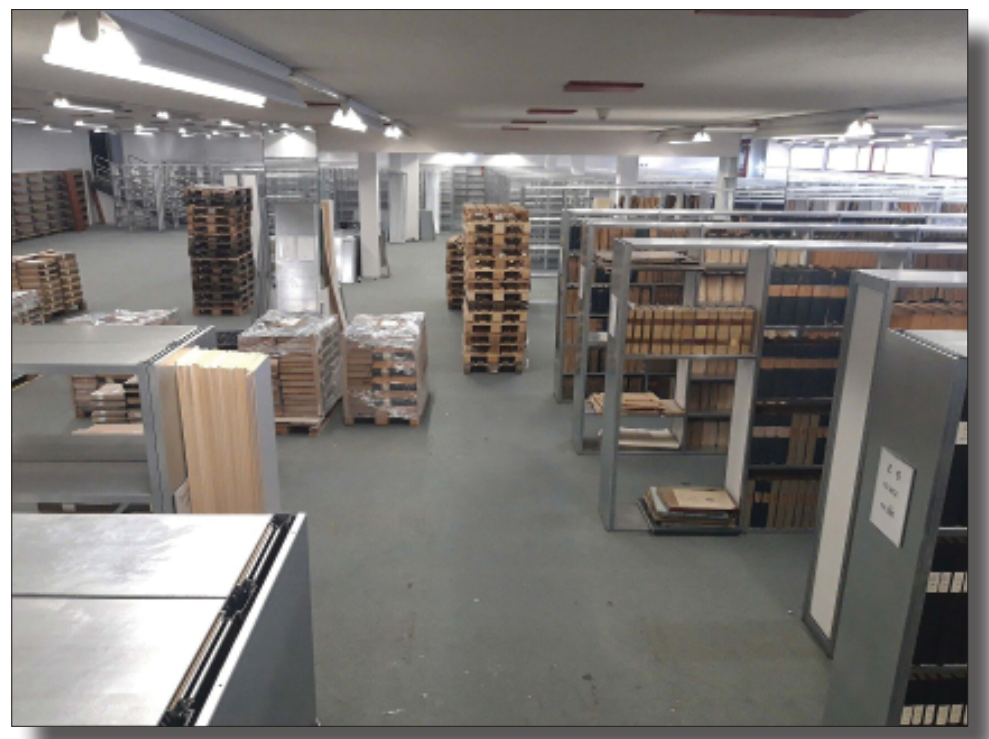

Abb. 1: Altes Depot der ULB Tirol in der Innsbrucker Bachlechner-Straße (Foto: ULB Tirol)

Erste Planungen und Gespräche für das Bedarfskonzept und eine entsprechende Berechnung erfolgten im Sommer 2017. Miteinbezogen wurde hier nicht nur eine Bestandsverlagerung unterschiedlichster bibliothekarischer Standorte (Hauptbibliothek, Bibliothekszentrum West, Fachbibliotheken Atrium und Rechtswissenschaft), sondern auch ein Platzbedarf seitens des Brenner- und des Universitätsarchivs. 
Diese Parameter waren wesentlich für die Berechnung der zu übersiedelnden Bestände, welche sich mit eigenen Magazins-Signaturen (keine RVK-Aufstellung) im Depot finden. Die Erstellung eines Belegplanes erbrachte einen Platzbedarf von $26.000 \mathrm{Ifm}$ mit hochgerechnet etwa 1 Million Medien sowie einem geplanten Regal- und Medien-Zuwachs für etwa 15 Jahre.

Die Anfertigung verschiedener Vertreter-Kärtchen (hauptsächlich im Zeitschriften-Bereich) ermöglichte eine konkretere Planung und berücksichtigte dabei auch passende Regalhöhen für unterschiedliche Formate sowie aktuell entlehnte Medien, bei denen man in etwa von einem Platzbedarf von ca. 5 bis $10 \mathrm{~cm}$ ausging.

50 Stück selbstkonstruierte Bücherroll-Regale erleichterten durch die schmale Bauweise den Abtransport aus den engen Magazins-Gängen und sorgten für eine schnellere und effektivere Absiedelung.

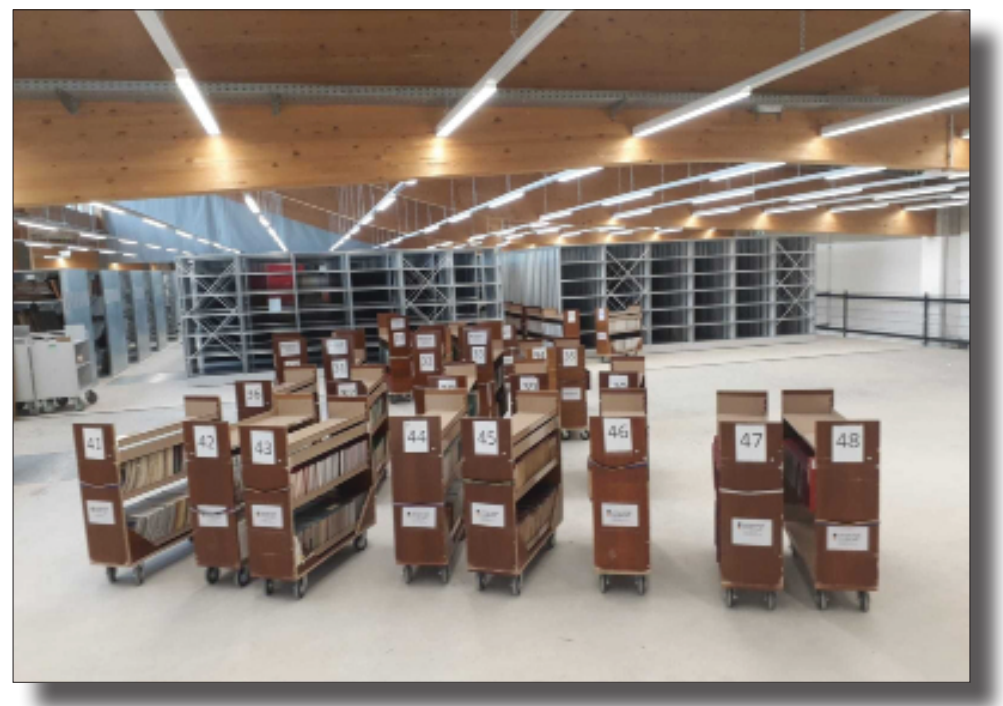

Abb. 2: Selbstkonstruierte Bücherwägen (Foto: ULB Tirol)

Die Durchführung der Transporte startete mit Februar 2020 und wurde durch die Corona-Pandemie kurzzeitig für etwa zwei Monate blockiert bzw. erschwert.

Bis Ende Juli konnten auf diese Weise Monografien und Zeitschriften komplett überführt werden, großformatige Medien bis Ende 2020. Der letzte Arbeitstag des "alten“ Magazins in der Innsbrucker BachlechnerStraße datiert übrigens mit 10. November 2020. 
Die Transportarbeiten wurden durch Mitarbeiter*innen aus unserer Logistik, Best-Practice-Kräften und weiteren Freiwilligen aus dem Team der ULB Tirol abgewickelt. Diese waren zuständig für die Be- und Entladung der Regale, Wägen und Paletten, den Fahr-Transport betreute ein eigens beauftragtes Unternehmen.

Parallel zu den Siedelungsmaßnahmen wurde der gesamte Ablauf sowie das benötigte Equipment fotografisch dokumentiert.

Die Lieferung der Bücher und Zeitschriften erfolgte mit Bücherrollregalen, Zeitungen und Großformate wurden mit Palettenwägen transportiert und zum Schutz auf Euro-Paletten foliert.

Pro Tag erfolgten durch das Transportunternehmen sieben bis acht Fahrten nach Gärberbach - mit je 17 Bücherrollregalen zu insgesamt etwa $51 \mathrm{lfm}$. Insgesamt ergaben sich hier über $330 \mathrm{lfm}$ an "Lieferleistung“ täglich.

Eine besondere Herausforderung stellte zudem die Aufrechterhaltung des Tagesgeschäftes (Medienbestellungen, Fahr- und Kurierdienste, BuchReparaturen etc.) während der Übersiedlungsarbeiten dar.

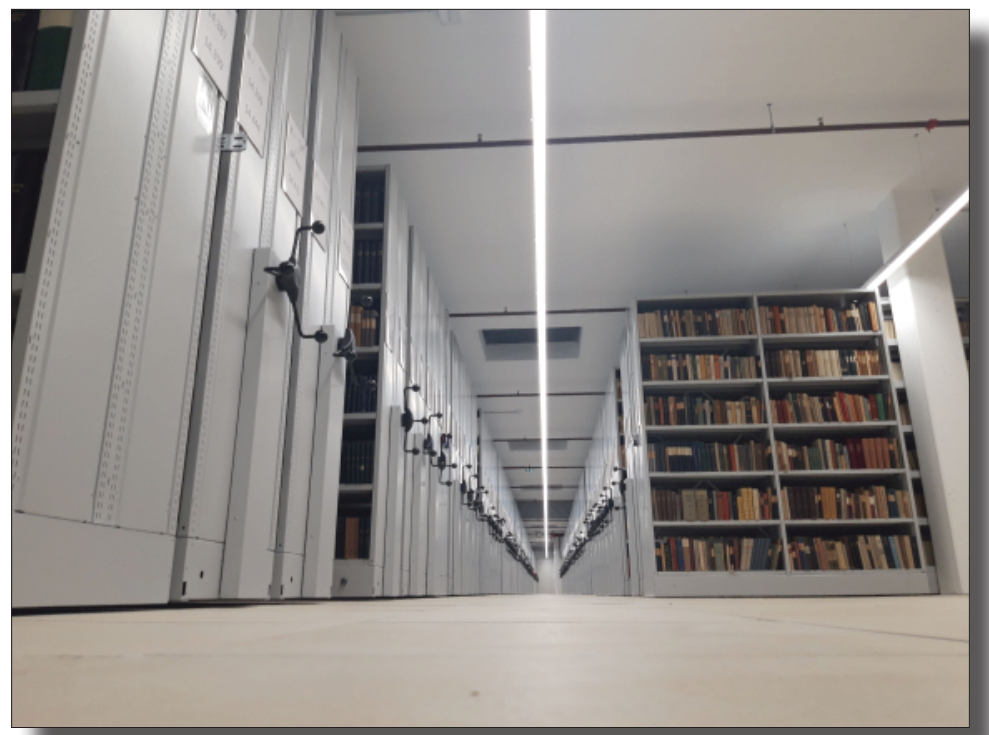

Abb. 3: Neues Zentraldepot der ULB Tirol in Gärberbach (Foto: ULB Tirol)

Abschließend geht ein herzliches Dankeschön an alle Mitarbeiter*innen, die dieses Projekt durch ihr großartiges Engagement ermöglicht haben. 
Christian Kössler

Universität Innsbruck,

Universitäts- und Landesbibliothek Tirol

E-Mail: christian.koessler@uibk.ac.at

DOI: https://doi.org/10.31263/voebm.v74i1.6241

(c) Christian Kössler

(c) (i)

Dieses Werk ist - exkl. einzelner Logos und Abbildungen - lizenziert unter einer Creative-Commons-Lizenz Namensnennung 4.0 International-Lizenz 